\title{
Circulating miR-375 Correlates With Response of Human Individuals to Short-Term Cold Exposure
}

Claudine Seeliger ( $\square$ claudine.seeliger@tum.de)

Institute of Nutritional Medicine, School of Medicine, Technical University of Munich

\section{Laura Aline Mengel}

Else Kröner-Fresenius Center for Nutritional Medicine, School of Life Sciences, Technical University of Munich

\section{Lise Buekens}

Else Kröner-Fresenius Center for Nutritional Medicine, School of Life Sciences, Technical University of Munich

\section{Alberto Mesas-Fernández}

Else Kröner-Fresenius Center for Nutritional Medicine, School of Life Sciences, Technical University of Munich

\section{Thomas Skurk}

ZIEL Institute for Food and Health

\section{Melina Claussnitzer}

Broad Institute of MIT and Harvard

\section{Hans Hauner}

Institute of Nutritional Medicine, School of Medicine, Technical University of Munich

\section{Research Article}

Keywords: serum miRNAs, miR-375, gender-dependent, non-shivering thermogenesis, cold exposure, biomarker

Posted Date: April 6th, 2021

DOI: https://doi.org/10.21203/rs.3.rs-382686/v1

License: (c) (i) This work is licensed under a Creative Commons Attribution 4.0 International License. Read Full License 
3

4 Claudine Seeliger 1,2,3, Laura Aline Mengel ${ }^{3}$, Lise Buekens ${ }^{3}$, Alberto Mesas-

5 Fernández ${ }^{3}$, Thomas Skurk ${ }^{2,3}$, Melina Claussnitzer ${ }^{4,5}$ and Hans Hauner 1,2,3

71 Institute of Nutritional Medicine, School of Medicine, Technical University of Munich, Munich,

8 Germany

92 ZIEL Institute for Food and Health, Technical University of Munich, Freising-Weihenstephan, Germany

3 Else Kröner-Fresenius Center for Nutritional Medicine, School of Life Sciences, Technical University of Munich, Freising-Weihenstephan, Germany

4 Broad Institute of MIT and Harvard, Cambridge, MA 02142, USA

5 Harvard Medical School, Harvard University, Boston, MA 02215, USA

Address correspondence and request for reprints to:

Dr. rer. nat. C. Seeliger

Else Kröner-Fresenius-Center for Nutritional Medicine

Chair of Nutritional Medicine

School of Life Sciences

Technical University of Munich

Gregor-Mendel-Str. 2

85354 Freising, Germany

Tel.: 08161/71-2006

Fax: 08161/71-2097

E-Mail: claudine.seeliger@tum.de

The authors declare no competing financial interests 
Cold-induced non-shivering thermogenesis (CIT) of the human body is currently discussed as an important contributor to energy metabolism, and miRNAs have recently been reported as key regulators of metabolism. Identifying the link of CIT and circulating microRNAs (miRNAs) in a large cohort of human individuals remains elusive to date. Here, we analyzed a set of 158 miRNAs in the serum of 97 female and 72 male healthy individuals before and after cold exposure (CE). Validating the results of a miRNA array, a significant down-regulation of miR-375 was measured in individuals after CIT $(P<0.0001)$. These changes went along with a significant negative correlation between miR-375 and the supraclavicular skin temperature $(P=0.012)$.

Additionally, the regulation of miR-375 was sex-dependent, with female individuals showing a significantly stronger decrease in expression of miR-375 under CIT compared to males. Such findings were already seen under thermoneutral conditions, where females display significant lower miR-375 expression levels $(P=0.015)$. Besides, the lower miR-375 expression levels were negatively correlated with the amount of visceral fat tissue in the female cohort $(P=0.0002)$.

This study identified miR-375 as a potential new sex-dependent marker for coldinduced thermogenesis.

KEY WORDS: serum miRNAs, miR-375, gender-dependent, non-shivering thermogenesis, cold exposure, biomarker 


\section{Introduction}

58 Recent studies suggest that activation of brown adipose tissue (BAT) may 59 increase energy expenditure by thermogenesis and affects total energy 60 homeostasis ${ }^{1}$. Mild cold exposure is known to activate BAT and, thereby, cold61 induced non-shivering thermogenesis (CIT) increases energy expenditure in 62 humans with residual BAT ${ }^{2}$. Due to its ability to oxidize glucose and lipids, BAT activation is reported to exert beneficial effects on glucose and lipid metabolism ${ }^{3,4}$. Furthermore, miRNAs have recently been implicated in metabolism in health and disease ${ }^{5}$

In this context, a few microRNAs (miRNAs) were identified to be involved in the thermogenesis regulation, although their role remains poorly understood because studies focus mainly on mice models or cell culture experiments ${ }^{6-8}$. miRNAs are non-coding, short RNA segments of approximately 22 nucleotides. They post-transcriptionally control mRNA expression by forming an RNAinduced silencing complex (RISC), which either leads to mRNA target cleavage or mRNA degradation and/or translation repression ${ }^{9,10}$. The miR-182-miR-203 cluster as well as miR-196a-5p and miR-328 were previously identified as positive regulators of brown/beige adipocyte development ${ }^{11-15}$. Opposite effects could be seen by stimulating cells with the panel miR-106b-93 or $\operatorname{miR}-125-5 p^{16,17}$

To date, little is known regarding miRNAs changes in serum in humans by BAT activation during cold exposure, taking sex, changes of body temperature and serum metabolites into account. The group of Chen et al. showed a negative correlation of exosome derived miR-92a with BAT activity in mice and a small 
81 cohort of human individuals undergoing $\mathrm{CE}^{8}$. This miRNA is also elevated in 82 obesity and decreased after bariatric surgery, correlating with metabolic 83 improvements ${ }^{18}$.

84 Recently our group reported that adult humans show high variability in their 85 increase in resting energy expenditure (REE) after a standardized mild 86 cold-exposure ${ }^{19}$ (Mengel, et al, submitted). The study also revealed an increase 87 in the expression of the browning marker gene CIDEA in female but not in male 88 participants. In the present study, we analyzed circulating miRNAs in a cohort 89 comprising 169 individuals exposed to moderate short-term cold to identify 90 specific miRNA signatures in response to the exposure. 
92

\section{Results}

Identification of differentially expressed miRNAs in the serum of individuals undergoing CIT using a miRNA array

We profiled the miRNA spectra via the human miRCURY LNA miRNA Focus PCR Panels before and after cold-exposure to identify response miRNAs in serum. Therefore, we used a combined miRNA sample from 12 males and in another run a combined miRNA sample of 12 females samples. We captured 158 different miRNAs across samples, while 11 miRNAs were not detectable. Among the most abundant miRNAs in serum, 5 miRNAs, including miR-22-3p, miR-99a-5p, miR-185-5p, miR-361-5p, and miR-375 were significantly dysregulated after CIT treatment (Fig. 1A). miR-361-5p displayed the most significant changes in the combined sample with a 5.5-fold difference compared to the samples before CIT. Raw data of the array are shown in Table S3.

\section{Specific circulating miRNAs under CE}

To validate the identified miRNAs as targets of CE exposure, we assayed the five miRNA identified in the discovery cohort as well miR-92a $a^{8}$ and miR-125 $5^{8,17}$, which has been previously implicated in brown/beige adipocyte function using qPCR on an independent validation sample set comprising serum samples from 169 individuals before and after CE. Among the selected miRNAs, miR-375 was predominantly down-regulated in the validation cohort (mean change - 0.922 to 1.042) (Fig. 1B). In contrast, all other assayed miRNAs displayed no significant changes during CIT. We further observed a significant negative correlation of miR-375 and the supraclavicular skin temperature $(r=-0.172, P=0.012$, Fig. 
1C), which is a proxy of BAT activity in humans ${ }^{20}$. Additionally, we observed significant changes in several blood parameters Table 1. Notable, lipolysisrelated clinical markers such as triglycerides, non-esterified fatty acids, and the adipokine adiponectin were significantly upregulated, whereas leptin displayed lower concentrations after CE.

\section{CE induced changes of miR-375 expression levels were different between} genders

To evaluate gender effects, we analyzed females and males separately and found that female individuals showed a significant stronger decrease of miR-375 gene expression levels in response to following $\mathrm{CE}(P=<0.0001$, Fig. 1D). In contrast, males displayed substantially smaller changes in response to CE $(P=0.0178)$. We observed differences between male and female individuals under thermoneutral conditions, with the levels of miR-375 in females were increased compared to males $(P=0.0147)$.

\section{miR-375 expression correlates with blood markers in females}

Down-regulation of miR-375 expression correlated significantly with the changes of free triiodothyronine levels $(P=0.0391)$ (Fig. 2A). Additionally, plasma insulin, as well as serum triglyceride levels, were negatively correlated with the changes of miR-375 during $\mathrm{CIT}(P=0.0347$ and $P=0.0453$, Fig. 2B-C). Furthermore, miR-375 under thermoneutral conditions was inversely correlated with the visceral fat content in female individuals $(r=-0.408, P=0.0002$, Fig. 
2D). The same correlation was seen for male participants $(r=-0.5404$, $P=<0.0001)$.

For assessment of miR-375 as a potential biomarker, we evaluate the changes of this miRNA in the female cohort. Thereby, relative changes of miR-375 were higher in individuals with higher expression of this miRNA at baseline, i.e. thermoneutrality $(P=<0.001$, Fig. 2E). We detected an $81 \%$ area under the receiver operating characteristic (ROC) curves for miR-375 values under thermoneutral conditions and the changes of miR-375 during CIT (Fig. 2F), with a threshold at -0.3995 .

\section{Functional enrichment analysis}

To analyze target mRNAs of the identified miR-375, we used the TarBASE v.8, a miRNA-gene interaction prediction tool. This search yielded 995 target mRNAs of this miRNA. Based on these potential target genes, GO analysis and KEGG pathway analysis were performed using the DIANA TOOLS to elucidate the biological functions of the target genes and the signaling pathways involved ${ }^{21}$. The GO analysis indicated that target genes were associated with ion binding, gene expression, Fc-epsilon receptor signaling pathway, molecular function, and neurotrophic TRK receptor signaling pathway, biological process, and cellular component regulation (Table S4). The results of KEGG analyses revealed target genes of miR-375 mainly with functions in protein processing of the endoplasmic reticulum, proteoglycans in cancer, viral carcinogenesis, and glycosaminoglycan biosynthesis (Fig. 3). 


\section{Discussion}

164 Cold-induced non-shivering thermogenesis recently received growing interest, as studies in humans suggested that the underlying activation of brown adipose tissue could play a significant role in energy homeostasis ${ }^{1}$. This study shows that miR-375 is associated with the response of participants to a moderate cold exposure.

Since their discovery in $1993^{22}$, miRNAs have been widely studied concerning their potential use for diagnostic purposes. Specific circulating miRNA profiles 
procedures, making the measurement of specific miRNAs easy to handle and cost-efficient. miR-375 could also be used as a monitory target molecule for cold exposure processes.

To date, some studies have indicated that specific miRNAs are involved in BAT activation during cold stimulation ${ }^{32,33}$. However, these studies mainly focused on identifying miRNAs in one cell type, such as immortalized brown adipocytes or mice mesenchymal stem cells. Also, a regulatory effect of certain miRNAs in specific animal models such as Ercc1\%, Dgcr8flox/flox, adiponectin-Cre transgenic mice, or Ppara ${ }^{-/}$was described ${ }^{34}$. For example, Oliverio et al. could identify miR-328 as a controller of brown adipose tissue differentiation and BAT function in mice ${ }^{13}$.

In the view of human individuals undergoing a mild cold exposure treatment, only exosomal expression changes of miR-92a and miR-122-5p were reported $^{8,35}$. Both groups found a negative association of these exo-miRNAs and BAT activation in mice and men. The human cohorts analyzed comprised a relatively small sample size of individuals or were sex restricted. In contrast, we analyzed circulating miRNAs because exosomal miRNA are mainly detected upon malignant conditions, and the majority of exo-miRs are not conserved in exosomes but rather bound to argonaut proteins ${ }^{36}$. Further, analyzing exo-miRs, the spectrum of detectable miRNAs is lower ${ }^{37}$. However, only expression levels of miR-375 were significantly changed during cold-stimulated non-shivering thermogenesis. Notable, this change was sex-dependent, suggesting a more robust response of females to CIT. Correlation of miR-375 with the 
210 supraclavicular skin temperature could may reflect its association with BAT activity.

miR-375, which was also down regulated after CIT, has not been previously implicated in BAT activation. In a study in individuals with or without diabetes, an up-regulation of miR-375 was in the diabetic group ${ }^{38}$. miR-375 has been shown to promote adipogenic differentiation by increasing mRNA levels of CEBPA and PPARG2 as well as by inducing adipocyte fatty acid-binding protein and triglyceride accumulation ${ }^{39}$. Besides, miR-375 was upregulated during the osteogenic differentiation of human adipose-derived mesenchymal stem cells ${ }^{40,41}$ highlighting its impact on mesenchymal cell differentiation capacity. Kraus, et al. identified miR-375 as an androgen regulated microRNA, showing an androgen mediated inhibition of miR-375 and the associated regulation of ADIPOR2 in differentiating human adipocytes. This could play an important role in the mechanism of the increase in visceral fat mass and the association with insulin resistance caused by testosterone deficiency ${ }^{42}$. It may be speculated that reduced levels of miR-375 may be related to adipocyte differentiation, shifting more cells in the beiging/browning process. Our additional target enrichment analysis did not provide a specific link between miR-375 and coldinduced thermogenesis, suggesting that miR375 might have a generic role on diverse cold induced processes in humans. Together, our findings raise interesting questions about the roles of this miRNA in view of the beiging/browning process of adipocytes and underline the need to understand the biological origins and functions of circulating miRNAs in this context. 
233 Overall, we could show that miR-375 was associated with the response or 234 non-response of CIT in a sex-dependent manner (Fig.4). The miRNA showed 235 significant sensitivity and specificity in distinguishing responders versus non236 responders. miR-375 represents a potential thermoneutral biomarker to 237 differentiate between CIT responder and non-responder individuals for basic 238 science and clinical applications, which can be measured with relative ease in 239 large cohorts of patients. 


\section{Methods}

242 Study approval: The local ethical review committee of the Faculty of Medicine of the Technical University of Munich approved the FREECE (Effect of the FTO-

Genotype on Resting Energy Expenditure after defined Cold Exposure) study (project number 236/16). The trial is also registered in the "Deutsches Register Klinischer Studien" (DRKS-ID: DRKS00010489). All participants provided written informed consent before any study procedures. All procedures were conducted according to the principles of the Declaration of Helsinki. The demographic data of the included individuals are presented in the supplemental material (Tables S1 and S2).

Short-term cold exposure: The participant was in a fasted state (12h) when the study started. First, measurements such as weight, height, waist and hip circumference were taken, as well as body composition data through bioimpedance (TANITA Body Composition Analyzer Type BC-418 MA, Tanita Europe $\mathrm{GmbH}$, Sindelfingen, Germany). Blood pressure and heart rate were measured, and the skin temperature was recorded via iButtons (Thermochron, Wisconsin, United States). Eight sensors were used to assess the overall skin temperature according to ISO $988620{ }^{43}$. One additional iButton was placed at the site of the supraclavicular area. We placed the participant in a supine position followed by a resting period of $15 \mathrm{~min}$, followed by $30 \mathrm{~min}$ of measurement of resting energy expenditure by indirect calorimetry (Cosmed Quark RMR 1.0, Fridolfing, Germany). Blood was taken from the participant, followed by the subsequent non-shivering cold exposure over two hours using water-contained thermic blankets (Maxi-Therm Lite, Cincinnati Sub-Zero 
265 Products, LLC). In the last $30 \mathrm{~min}$ of $\mathrm{CE}$, resting metabolic rate by indirect calorimetry was measured again, followed by evaluation of the blood pressure and heart rate. Immediately the second blood drawing took place. For detailed description of the cooling protocol, see relevant publication ${ }^{19}$.

Blood sample analysis: Blood samples before and after cold exposure were analyzed by a certified laboratory (Synlab, Munich, Germany) to obtain the parameters free triiodothyronine $\left(T_{3}\right)$, C-reactive protein, and total triglycerides in serum. Plasma non-esterified fatty acids (Wako Chemicals, Neuss, Germany), insulin (DRG Instruments, Marburg, Germany), leptin, and total adiponectin (R\&D, Wiesbaden, Germany) were analyzed with commercially available relevant ELISA kits as recommended by the company, respectively.

Sample processing and miRNA extraction: Serum samples frozen at $-80^{\circ} \mathrm{C}$ were thawed on ice and centrifuged at $16,000-\mathrm{x} \mathrm{g}$ for $5 \mathrm{~min}$ at $4^{\circ} \mathrm{C}$. miRNA was extracted from $200 \mu \mathrm{l}$ serum using TRIzol ${ }^{\mathrm{TM}}$ Reagent (Thermo Fisher Scientific, Massachusetts, USA) and the miRNeasy Serum/Plasma Advanced Kit, according to the manufacturer's recommendations (Qiagen, Hilden, Germany). RNA was precipitated with $900 \mu \mathrm{l}$ ethanol absolute, triple washed with wash solution, followed by RNA elution in $20 \mu \mathrm{l}$ nuclease-free water, and storage at $-80^{\circ} \mathrm{C}$. The amount and integrity of isolated miRNA were estimated via gel electrophoresis using a Small RNA chip examined by a Bioanalyzer device (Agilent Technologies, California, USA).

qPCR analysis: Subsequently, two $\mu$ miRNA with the addition of the spike-in control UniSp6 for considering enzyme efficiency was reversely transcribed into 
cDNA using the miRCURY LNA RT Kit (Qiagen, Hilden, Germany). The reaction was incubated at $42^{\circ} \mathrm{C}$ for $60 \mathrm{~min}$ and then heat-inactivated at $95^{\circ} \mathrm{C}$ for 5 min. cDNA samples were stored at $-20^{\circ} \mathrm{C}$. Real-Time quantitative PCR (RTqPCR) was conducted using custom 384 well panels (4titude, Wotton, UK). For RT-qPCR analysis, cDNA samples were diluted 20 -fold, and $4 \mu$ l were used in individual $10 \mu \mathrm{l}$ PCR reactions using miRCURY Sybr Green Kit and LNAenhanced miRNA primer assays together with UniSp6 as a spike-in control (Qiagen, Hilden, Germany). PCR conditions were $95^{\circ} \mathrm{C}$ for $2 \mathrm{~min}, 50$ cycles of denaturation (95C, 10s) and annealing (56C, 60s), and melting curve analysis on LC480 Real-Time PCR system (Roche, Basel, Switzerland). To calculate the $\mathrm{C}_{\mathrm{q}}$-values, the second derivative method was used.

miRNAs array analysis: We profiled miRNA spectra from two pooled serum groups, including one pool of 12 males and another of 12 female samples, to identify regulated miRNAs during cold induced thermogenesis. In total, 169 different miRNAs were profiled by the human miRCURY LNA miRNA Focus PCR Panels YAHS-106YG-2 (Qiagen, Hilden, Germany). Expression levels of were determined by the cycle number via qPCR. Levels were normalized to the internal reference genes using the $2^{-\Delta \Delta C t}$ method ${ }^{44}$. Afterward, the fold-change before and after cold exposure regarding the expression of specific miRNAs was calculated.

miRNA expression analysis: The set of seven circulating miRNAs, selected based on the previous array results and in the literature described miR-92a-5p as well as miR-125a-5p, were analyzed in the 169 serum samples before and after cold-induced thermogenesis $8,17,45,46$. For identifying hemolysis samples, 
313 the level of miR-451a (microRNA highly abundant in red blood cells) was assessed in all isolated samples. The concentration of miR-451 was significantly lower in all included samples compared to our internal positive control $(1 \mathrm{ml}$ of $9 \mathrm{ml}$ blood mixed with $1 \mathrm{ml}$ Lysis buffer $(155 \mathrm{mM} \mathrm{NH} 4 \mathrm{Cl}, 5.7 \mathrm{mM}$ K2HPO4, 0.1 mM EDTA x 2H2O, pH 7.4), incubated 10min RT, both separately centrifuged $10 \mathrm{~min}, 12,000 \mathrm{~g}, 4^{\circ} \mathrm{C}$, mixing serum and $1 \mathrm{ml}$ lysed serum centrifuged $10 \mathrm{~min}, 16,000 \mathrm{~g}, 4^{\circ} \mathrm{C}$, Cutoff $\mathrm{Cq}$ value $\left.=15\right)$. Following a screening of potential reference genes, miR-30e-5p and let-7i-5p shown stable expression levels serving in the validation assays for normalization.

Statistical analyses: Results are given (after age and BMI adjustment as well as logarithmic transformation) as scatter dot plots, mean with standard deviation $( \pm \mathrm{SD}$ ). All data were non-Gaussian distributed (Kolmogorov-Smirnov test $P<0.05)$. An one-tailed unpaired Mann Whitney test was used to determine the significance of differential results from two groups. For data from before and after cold-stimulated non-shivering thermogenesis, Wilcoxon signed-rank test one-tailed was used. For all statistical analysis, GraphPad Prism version 5.02 was used (Graph Pad Software, San Diego, USA). To determine the diagnostic utility of serum miRNA, we used ROC curves. The $P$-value tests the null hypothesis that the area under the curve equals 0.50 . The cutoff points with the highest sensitivity and specificity were determined. $P<0.05$ was taken as a minimum level of significance. 
3361 Cypess, A. M. \& Kahn, C. R. The role and importance of brown adipose tissue in energy 337 homeostasis. Curr Opin Pediatr 22, 478-484, doi:10.1097/MOP.0b013e32833a8d6e (2010).

Nishimura, T. et al. Seasonal variation of non-shivering thermogenesis (NST) during mild cold exposure. J Physiol Anthropol 34, 11, doi:10.1186/s40101-015-0051-9 (2015).

Jeremic, N., Chaturvedi, P. \& Tyagi, S. C. Browning of White Fat: Novel Insight Into Factors, Mechanisms, and Therapeutics. doi: $10.1002 /$ jcp. 25450 (2017).

Peirce, V. \& Vidal-Puig, A. Regulation of glucose homoeostasis by brown adipose tissue. Lancet Diabetes Endocrinol 1, 353-360, doi:10.1016/S2213-8587(13)70055-X (2013).

Wang, L. et al. A MicroRNA Linking Human Positive Selection and Metabolic Disorders. Cell 183, 684-701 e614, doi:10.1016/j.cell.2020.09.017 (2020).

Lemecha, M. et al. Improved glucose metabolism by Eragrostis tef potentially through beige adipocyte formation and attenuating adipose tissue inflammation. PLoS One 13, e0201661, doi:10.1371/journal.pone.0201661 (2018).

Ng, R. et al. miRNA-32 Drives Brown Fat Thermogenesis and Trans-activates Subcutaneous White Fat Browning in Mice. Cell Rep 19, 1229-1246, doi:10.1016/j.celrep.2017.04.035 (2017).

Chen, Y. et al. Exosomal microRNA miR-92a concentration in serum reflects human brown fat activity. Nature communications 7, 11420-11420, doi:10.1038/ncomms11420 (2016).

Shimoni, Y. et al. Regulation of gene expression by small non-coding RNAs: a quantitative view. Mol Syst Biol 3, 138, doi:10.1038/msb4100181 (2007).

Pfeifer, A. \& Lehmann, H. Pharmacological potential of RNAi--focus on miRNA. Pharmacol Ther 126, 217-227, doi:10.1016/j.pharmthera.2010.03.006 (2010).

Kim, H. J. et al. MicroRNAs are required for the feature maintenance and differentiation of brown adipocytes. Diabetes 63, 4045-4056, doi:10.2337/db14-0466 (2014).

Mori, M., Nakagami, H., Rodriguez-Araujo, G., Nimura, K. \& Kaneda, Y. Essential role for miR-196a in brown adipogenesis of white fat progenitor cells. PLoS Biol 10, e1001314, doi:10.1371/journal.pbio.1001314 (2012).

Oliverio, M. et al. Dicer1-miR-328-Bace1 signalling controls brown adipose tissue differentiation and function. Nat Cell Biol 18, 328-336, doi:10.1038/ncb3316 (2016).

14 Meakin, P. J. et al. Reduction in BACE1 decreases body weight, protects against diet-induced obesity and enhances insulin sensitivity in mice. Biochem $J$ 441, 285-296, doi:10.1042/BJ20110512 (2012).

Sun, L. et al. Mir193b-365 is essential for brown fat differentiation. Nat Cell Biol 13, 958-965, doi:10.1038/ncb2286 (2011).

Wu, Y. et al. Identification of miR-106b-93 as a negative regulator of brown adipocyte differentiation. Biochem Biophys Res Commun 438, 575-580, doi:10.1016/j.bbrc.2013.08.016 (2013).

Giroud, M. et al. miR-125b affects mitochondrial biogenesis and impairs brite adipocyte formation and function. Mol Metab 5, 615-625, doi:10.1016/j.molmet.2016.06.005 (2016). 
18 Cereijo, R. et al. Elevated Levels of Circulating miR-92a Are Associated with Impaired Glucose Homeostasis in Patients with Obesity and Correlate with Metabolic Status After Bariatric Surgery. Obes Surg, doi:10.1007/s11695-019-04104-y (2019).

19 Mengel, L. A. et al. Gender Differences in the Response to Short-term Cold Exposure in Young Adults. The Journal of clinical endocrinology and metabolism 105, doi:10.1210/clinem/dgaa110 (2020).

20 van der Lans, A. A., Vosselman, M. J., Hanssen, M. J., Brans, B. \& van Marken Lichtenbelt, W. D. Supraclavicular skin temperature and BAT activity in lean healthy adults. J Physiol Sci 66, 77-83, doi:10.1007/s12576-015-0398-z (2016).

21 Vlachos, I. S. et al. DIANA-miRPath v3.0: deciphering microRNA function with experimental support. Nucleic Acids Res 43, W460-466, doi:10.1093/nar/gkv403 (2015).

22 Lee, R. C., Feinbaum, R. L. \& Ambros, V. The C. elegans heterochronic gene lin-4 encodes small RNAs with antisense complementarity to lin-14. Cell 75, 843-854, doi:10.1016/00928674(93)90529-y (1993).

23 Rupaimoole, R. \& Slack, F. J. MicroRNA therapeutics: towards a new era for the management of cancer and other diseases. Nat Rev Drug Discov 16, 203-222, doi:10.1038/nrd.2016.246 (2017).

24 Seeliger, C. et al. Five freely circulating miRNAs and bone tissue miRNAs are associated with osteoporotic fractures. J Bone Miner Res 29, 1718-1728, doi:10.1002/jbmr.2175 (2014).

25 Seeliger, C., Balmayor, E. R. \& van Griensven, M. miRNAs Related to Skeletal Diseases. Stem Cells Dev 25, 1261-1281, doi:10.1089/scd.2016.0133 (2016).

26 Adachi, T. et al. Plasma microRNA 499 as a biomarker of acute myocardial infarction. Clinical chemistry 56, 1183-1185, doi:10.1373/clinchem.2010.144121 (2010).

27 Zhang, L. et al. Clinical significance of circulating microRNAs as diagnostic biomarkers for coronary artery disease. Journal of cellular and molecular medicine, doi:10.1111/jcmm. 14802 (2019).

28 Zaiou, M., El Amri, H. \& Bakillah, A. The clinical potential of adipogenesis and obesity-related microRNAs. Nutr Metab Cardiovasc Dis 28, 91-111, doi:10.1016/j.numecd.2017.10.015 (2018).

29 Mononen, N. et al. Whole blood microRNA levels associate with glycemic status and correlate with target mRNAs in pathways important to type 2 diabetes. Sci Rep 9, 8887, doi:10.1038/s41598-019-43793-4 (2019).

30 Virtanen, K. A. et al. Human adipose tissue glucose uptake determined using [(18)F]-fluorodeoxy-glucose $([(18) \mathrm{F}] \mathrm{FDG})$ and PET in combination with microdialysis. Diabetologia 44, 2171-2179, doi:10.1007/s001250100026 (2001).

31 van Marken Lichtenbelt, W. D. et al. Cold-activated brown adipose tissue in healthy men. $N$ Engl J Med 360, 1500-1508, doi:10.1056/NEJMoa0808718 (2009).

32 Yang, N. et al. Tumor necrosis factor alpha suppresses the mesenchymal stem cell osteogenesis promoter miR-21 in estrogen deficiency-induced osteoporosis. Journal of bone and mineral research : the official journal of the American Society for Bone and Mineral Research 28, 559573, doi:10.1002/jbmr.1798 (2013).

33 Wang, W. T., Zhao, Y. N., Han, B. W., Hong, S. J. \& Chen, Y. Q. Circulating microRNAs identified in a genome-wide serum microRNA expression analysis as noninvasive biomarkers for endometriosis. The Journal of clinical endocrinology and metabolism 98, 281-289, doi:10.1210/jc.2012-2415 (2013). 
Pal, A. S. \& Kasinski, A. L. Animal Models to Study MicroRNA Function. Adv Cancer Res 135, 53-118, doi:10.1016/bs.acr.2017.06.006 (2017).

Okamatsu-Ogura, Y. et al. Association of circulating exosomal miR-122 levels with BAT activity in healthy humans. Sci Rep 9, 13243, doi:10.1038/s41598-019-49754-1 (2019).

Bhome, R. et al. Exosomal microRNAs (exomiRs): Small molecules with a big role in cancer. Cancer Lett 420, 228-235, doi:10.1016/j.canlet.2018.02.002 (2018).

Turchinovich, A., Weiz, L., Langheinz, A. \& Burwinkel, B. Characterization of extracellular circulating microRNA. Nucleic Acids Res 39, 7223-7233, doi:10.1093/nar/gkr254 (2011).

Higuchi, C. et al. Identification of circulating miR-101, miR-375 and miR-802 as biomarkers for type 2 diabetes. Metabolism 64, 489-497, doi:10.1016/j.metabol.2014.12.003 (2015).

Ling, H. Y. et al. MicroRNA-375 promotes 3T3-L1 adipocyte differentiation through modulation of extracellular signal-regulated kinase signalling. Clin Exp Pharmacol Physiol 38, 239-246, doi:10.1111/j.1440-1681.2011.05493.x (2011).

Chen, S. et al. Exosomes derived from miR-375-overexpressing human adipose mesenchymal stem cells promote bone regeneration. Cell Prolif 52, e12669, doi:10.1111/cpr.12669 (2019).

Chen, S., Zheng, Y., Zhang, S., Jia, L. \& Zhou, Y. Promotion Effects of miR-375 on the Osteogenic Differentiation of Human Adipose-Derived Mesenchymal Stem Cells. Stem Cell Reports 8, 773-786, doi:10.1016/j.stemcr.2017.01.028 (2017).

42 Kraus, M. et al. Inhibition of adipogenic differentiation of human SGBS preadipocytes by androgen-regulated microRNA miR-375. Mol Cell Endocrinol 414, 177-185, doi:10.1016/j.mce.2015.07.026 (2015).

International, Standards \& Organization. (Geneva, 2004).

Bustin, S. A. et al. The MIQE guidelines: minimum information for publication of quantitative real-time PCR experiments. Clinical chemistry 55, 611-622, doi:10.1373/clinchem.2008.112797 (2009).

Chen, Y. et al. Exosomal microRNA miR-92a concentration in serum reflects human brown fat activity. Nat Commun 7, 11420, doi:10.1038/ncomms11420 (2016). 
Author contributions Statement

C.S., M.C. and H.H. designed the research. C.S., L.B., L.A.M. and A.M. the manuscript and is responsible for the integrity of the data analysis.

Acknowledgements decision to publish, or manuscript preparation. We would like to thank Dr. Lynne Stecher for assisting with data analysis.

472 manner. Supplemental data have been included with this submission. 


\section{Figure Legends}

Fig. 1. miRNA expression pattern in humans after acute cold exposure is gender dependent. (A) Scatter plot analysis between logarithmic expression levels $\left(\mathrm{C}_{\mathrm{q}}\right)$ and fold-change of miRNAs after cold-induced thermogenesis measured via microarray. Labelled in black are the chosen miRNAs for validation analysis. (B) Serum samples analysis of 169 individuals undergoing cold-stimulated non-shivering thermogenesis revealed significant downregulation of miR-375 (Wilcoxon signed-rank test, one-tailed, $P=<0.0001$ ). (C) The total cohort has shown a significant correlation of miR-375 changes and the supraclavicular temperature (Spearman $r=-0.172, P=0.012$ ) (D) miRNA expression of miR-375 were sex-dependent (Wilcoxon signed-rank test, onetailed, $P<0.001, P=0.018)$. Under thermoneutral conditions, females displayed significantly higher miR-375 expression values (Mann Whitney test, one-tailed, $P=0.015)$. Data are normalized to relevant housekeeping genes, age and BMI adjusted, $\log _{10}$ transferred and shown as mean $\pm S D$, CIT= cold-induced nonshivering thermogenesis, $\mathrm{TN}=$ thermoneutral, $\mathrm{Tsupra}=$ supraclavicular temperature $\left({ }^{\circ} \mathrm{C}\right)$

Fig. 2. Female individuals showed significant changes with important blood values. (A-C) Changes of miR-375 during CE were significantly and negatively correlated with free triiodothyronine (Spearman $r=-0.181, P=0.0391$ ), TGs (Spearman $r=-0.175, P=0.0453$ ) and insulin level (Spearman $r=-0.186$, $P=0.0347$ ). (D) Under thermoneutral conditions, miR-375 significantly correlated 
with visceral adipose tissue content of the body in female individuals (Spearman $\mathrm{r}=-0.408, P=0.0002) .(\mathrm{E}) \mathrm{miR}-375 \mathrm{TN}$ values strongly correlated with delta values of miR-375 during CE (Spearman $r=-0.451, P=<0.001$ ). (F) Diagnostic value of miR-375 under a thermoneutral condition with calculated delta values are displayed using a related receiver operating characteristic (ROC) curve. Data are normalized to relevant housekeeping genes, age and BMI adjusted, $\log _{10}$ transferred and shown as mean $\pm S D, A U C=$ area under the curve, conf. inter= confidence interval, $\mathrm{CIT}=$ cold-induced non-shivering thermogenesis, free trijodothyronine $(\mathrm{pmol} / \mathrm{l}), \mathrm{TGs}=$ triglycerides $(\mathrm{mg} / \mathrm{dl})$, insulin $(\mathrm{ng} / \mathrm{ml})$, $\mathrm{TN}=$ thermoneutral condition, visceral adipose tissue (I), $\mathrm{CE}=$ cold exposure

Fig. 3. Associated RNA target pathways of miR-375 Results of KEGG analyses revealed target genes of miR-375 mainly with functions in protein processing of the endoplasmic reticulum, proteoglycans in cancer, viral carcinogenesis, and glycosaminoglycan biosynthesis.

Fig. 4. Summarized findings miR-375 changes in the serum of 97 female and 72 male healthy individuals before and after cold exposure (CE). miR-375 was associated with the response or non-response of CIT in a sex-dependent manner. 
Tables

\begin{tabular}{|c|c|c|c|c|c|c|c|}
\hline & & & $\mathrm{N}$ & & $E$ & & \\
\hline Parameter $(\Delta)$ & $\mathrm{N}$ & Mean & Std. Dev. & Mean & Std. Dev. & Regulation & $\begin{array}{c}\text { Wilcoxon } \\
\text { test } \\
\text { p-value }\end{array}$ \\
\hline $\operatorname{REE}(\mathrm{Kcal} / \mathrm{d})$ & 169 & 1609 & 294.9 & 1726 & 360.4 & $\uparrow$ & $<0.0001$ \\
\hline Resperatory quotient & 169 & 0.782 & 0.074 & 0.748 & 0.066 & $\downarrow$ & $<0.0001$ \\
\hline $\mathrm{CRP}(\mathrm{mg} / \mathrm{l})$ & 168 & 0.238 & 0.424 & 0.252 & 0.445 & $\uparrow$ & $<0.0001$ \\
\hline HR (beats/min) & 169 & 68.560 & 11.820 & 62.570 & 10.330 & $\downarrow$ & $<0.0001$ \\
\hline $\begin{array}{l}\text { Systolic blood preasure } \\
(\mathrm{mmHg})\end{array}$ & 169 & 116.000 & 12.180 & 120.000 & 12.120 & $\uparrow$ & $<0.0001$ \\
\hline $\begin{array}{l}\text { Diastolic blood preasure } \\
\text { (mmHg) }\end{array}$ & 169 & 75.850 & 7.819 & 81.210 & 7.870 & $\uparrow$ & $<0.0001$ \\
\hline $\mathrm{Glc}(\mathrm{mg} / \mathrm{dl})$ & 167 & 91.900 & 8.197 & 85.900 & 9.108 & $\downarrow$ & $<0.0001$ \\
\hline $\mathrm{TG}(\mathrm{mg} / \mathrm{dl})$ & 168 & 82.930 & 39.950 & 92.000 & 41.140 & $\uparrow$ & $<0.0001$ \\
\hline fT3 (pmol/l) & 168 & 3.281 & 0.3944 & 3.184 & 0.4061 & $\downarrow$ & $<0.0001$ \\
\hline $\operatorname{NEFA}(\mu \mathrm{mol} / \mathrm{l})$ & 168 & 292.300 & 127.600 & 521.800 & 219.300 & $\uparrow$ & $<0.0001$ \\
\hline Insulin (ng/ml) & 168 & 9.803 & 11.340 & 9.484 & 10.480 & $\downarrow$ & \\
\hline Adiponectin $(\mu \mathrm{g} / \mathrm{ml})$ & 168 & 7.667 & 5.153 & 8.730 & 6.836 & $\uparrow$ & $<0.0001$ \\
\hline Leptin (ng/ml) & 168 & 12.010 & 17.280 & 8.969 & 14.220 & $\downarrow$ & $<0.0001$ \\
\hline Tsupra $\left({ }^{\circ} \mathrm{C}\right)$ & 168 & 35.690 & 0.594 & 35.650 & 0.796 & $\rightarrow$ & \\
\hline Tskin $\left({ }^{\circ} \mathrm{C}\right)$ & 167 & 33.950 & 0.467 & 30.380 & 1.328 & $\downarrow$ & $<0.0001$ \\
\hline
\end{tabular}

Table1: Analysed parameters revealed significant changes for Resting Energy Expenditure (REE), FGF21, NEFA, Glc, Tsupra, and HR in individuals during acute cold exposure.

$\mathrm{CE}=$ cold exposure, $\mathrm{Glc}=$ fasting glucose, $\mathrm{HR}=$ heart rate, NEFA = Non-esterified Fatty Acids, REE = Resting Energy Expenditure, $\mathrm{RQ}=$ respiratory quotient, $\mathrm{TN}=$ thermoneutrality, $\mathrm{TG} s=$ triglycerides, $\mathrm{Tsupra}=$ supraclavicular temperature 

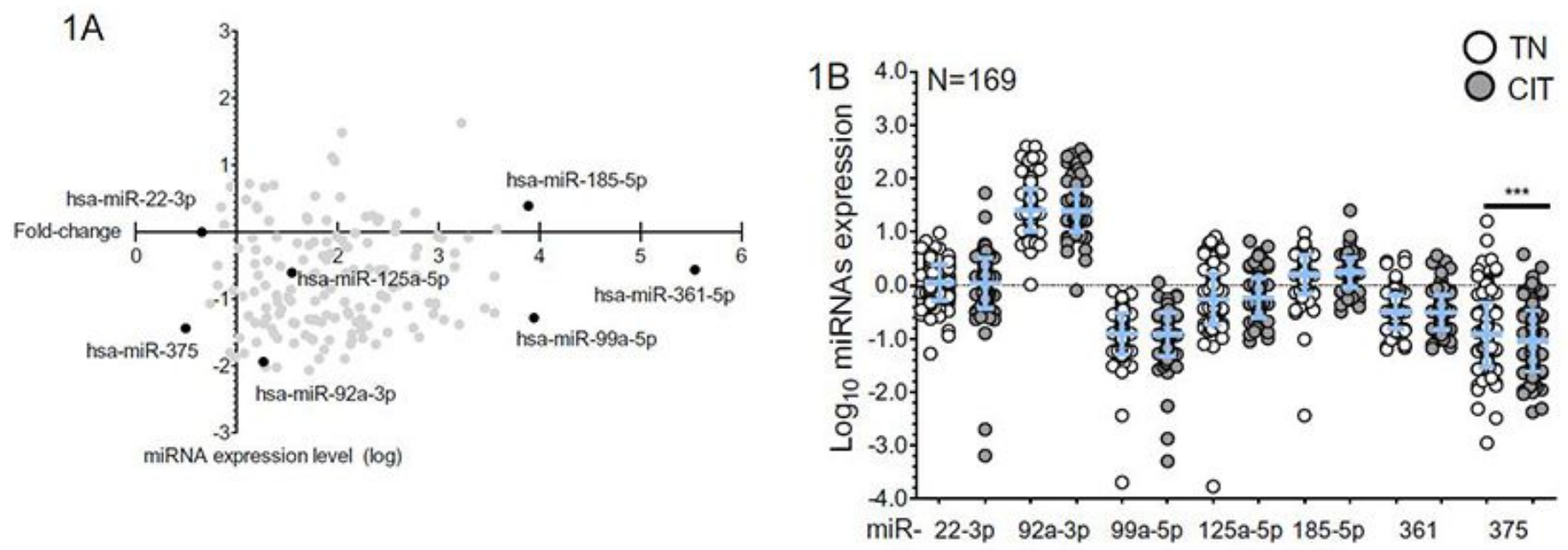

miR-375

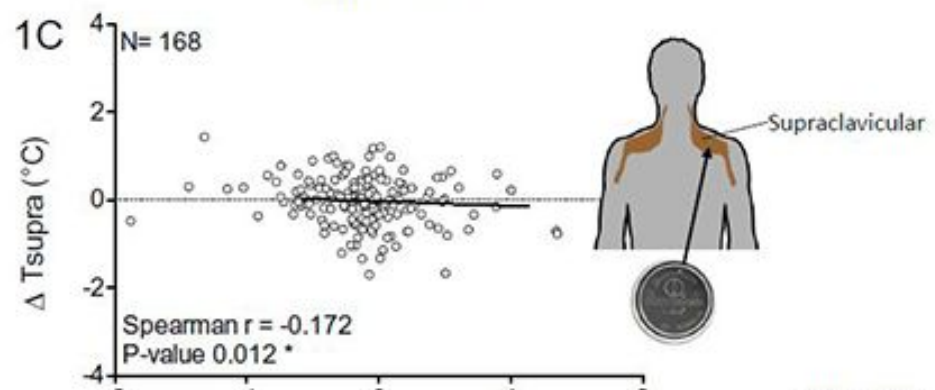

$\operatorname{miR}-375$

$\Delta \log _{10} \operatorname{miR}-375$ expression

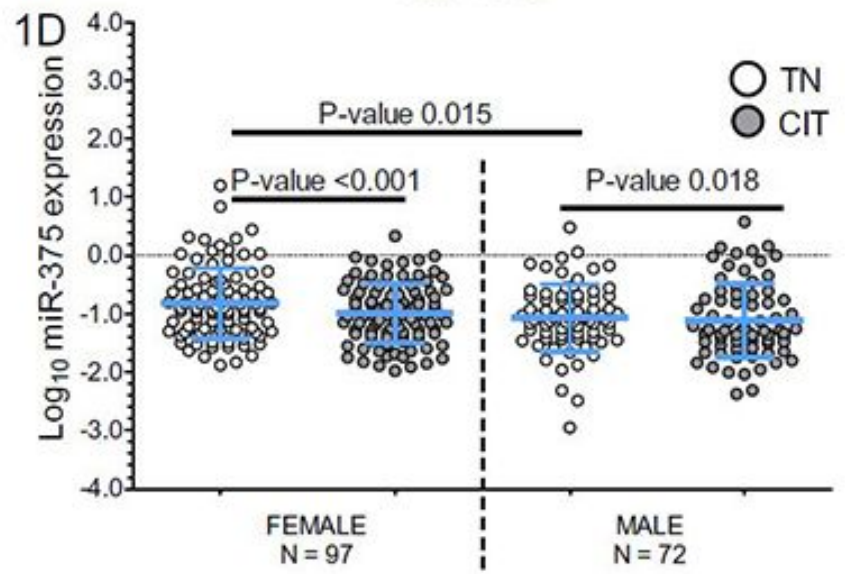

\section{Figure 1}

miRNA expression pattern in humans after acute cold exposure is gender dependent. (A) Scatter plot analysis between logarithmic expression levels $(\mathrm{Cq})$ and fold-change of miRNAs after cold-induced thermogenesis measured via microarray. Labelled in black are the chosen miRNAs for validation analysis. 
(B) Serum samples analysis of 169 individuals undergoing cold-stimulated non-shivering thermogenesis revealed significant downregulation of miR-375 (Wilcoxon signed-rank test, one-tailed, $P=<0.0001$ ). (C) The total cohort has shown a significant correlation of miR-375 changes and the supraclavicular temperature (Spearman $r=-0.172, P=0.012$ ) (D) miRNA expression of miR-375 were sex-dependent (Wilcoxon signed-rank test, one tailed, $\mathrm{P}<0.001, \mathrm{P}=0.018$ ). Under thermoneutral conditions, females displayed significantly higher miR-375 expression values (Mann Whitney test, one-tailed, $P=0.015$ ). Data are normalized to relevant housekeeping genes, age and BMI adjusted, log10 transferred and shown as mean $\pm \mathrm{SD}, \mathrm{CIT}=$ cold-induced non shivering thermogenesis, $\mathrm{TN}=$ thermoneutral, Tsupra = supraclavicular temperature $\left({ }^{\circ} \mathrm{C}\right)$

Figure2A-D
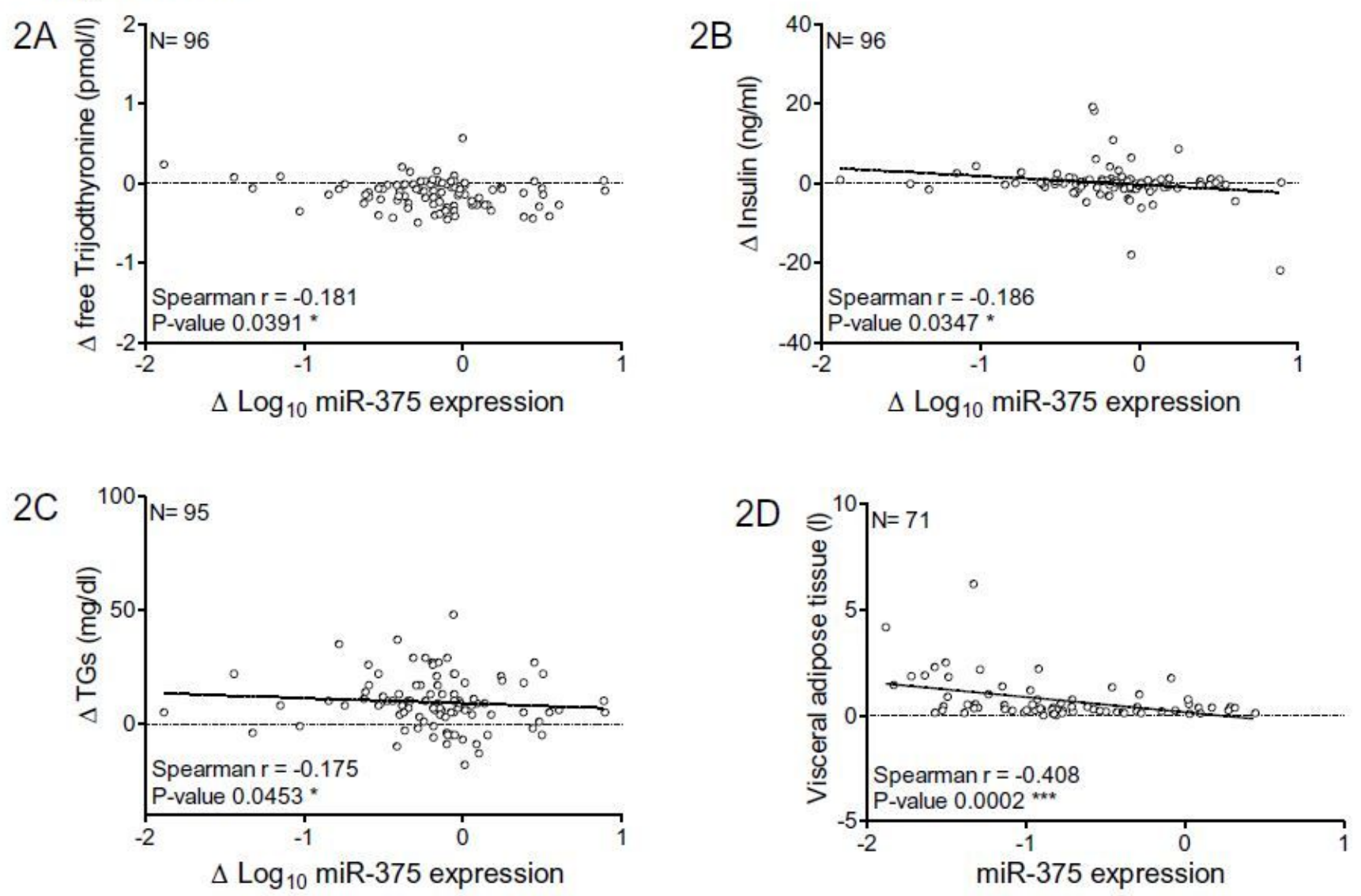

\section{Figure 2}

Female individuals showed significant changes with important blood values. (A-C) Changes of miR-375 during CE were significantly and negatively correlated with free triiodothyronine (Spearman $r=-0.181$, $P=0.0391$ ), TGs (Spearman $r=-0.175, P=0.0453$ ) and insulin level (Spearman $r=-0.186, P=0.0347)$. (D) Under thermoneutral conditions, miR-375 significantly correlated with visceral adipose tissue content of the body in 502 female individuals (Spearman $r=-0.408, P=0.0002$ ). (E) miR-375 TN values strongly correlated with delta values of miR-375 during CE (Spearman $r=-0.451, P=<0.001$ ). ( $F$ ) Diagnostic value of miR-375 under a thermoneutral condition with calculated delta values are displayed using a related receiver operating characteristic $(\mathrm{ROC})$ curve. Data are normalized to relevant housekeeping genes, age 
and $\mathrm{BMI}$ adjusted, $\log 10$ transferred and shown as mean $\pm \mathrm{SD}, \mathrm{AUC}=$ area under the curve, conf. inter= confidence interval, CIT= cold-induced non-shivering thermogenesis, free trijodothyronine $(\mathrm{pmol} / \mathrm{l}), \mathrm{TGs}=$ triglycerides $(\mathrm{mg} / \mathrm{dl})$, insulin $(\mathrm{ng} / \mathrm{ml}), \mathrm{TN}=$ thermoneutral condition, visceral adipose tissue $(\mathrm{l}), \mathrm{CE}=$ cold exposure

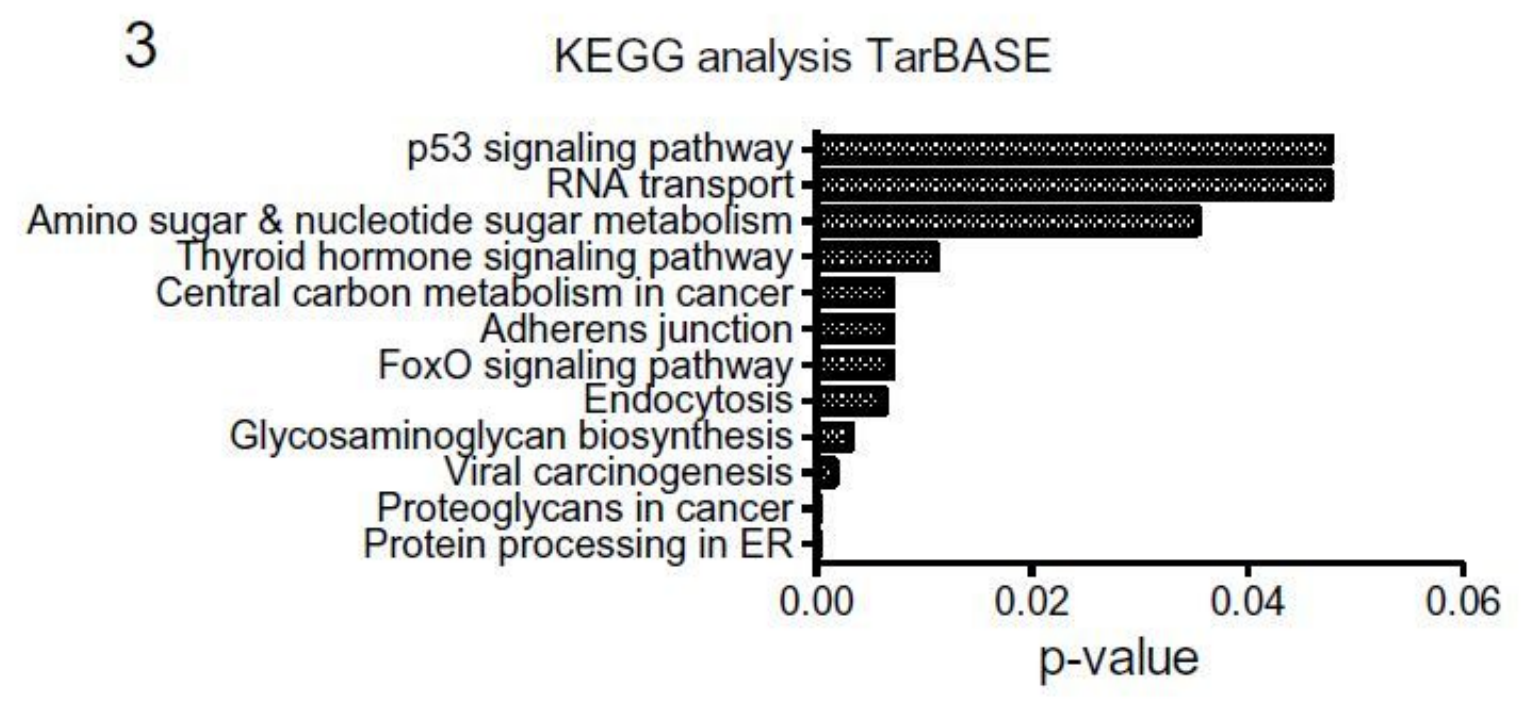

Figure 3

Associated RNA target pathways of miR-375 Results of KEGG analyses revealed target genes of miR-375 mainly with functions in protein processing of the endoplasmic reticulum, proteoglycans in cancer, viral carcinogenesis, and glycosaminoglycan biosynthesis. 


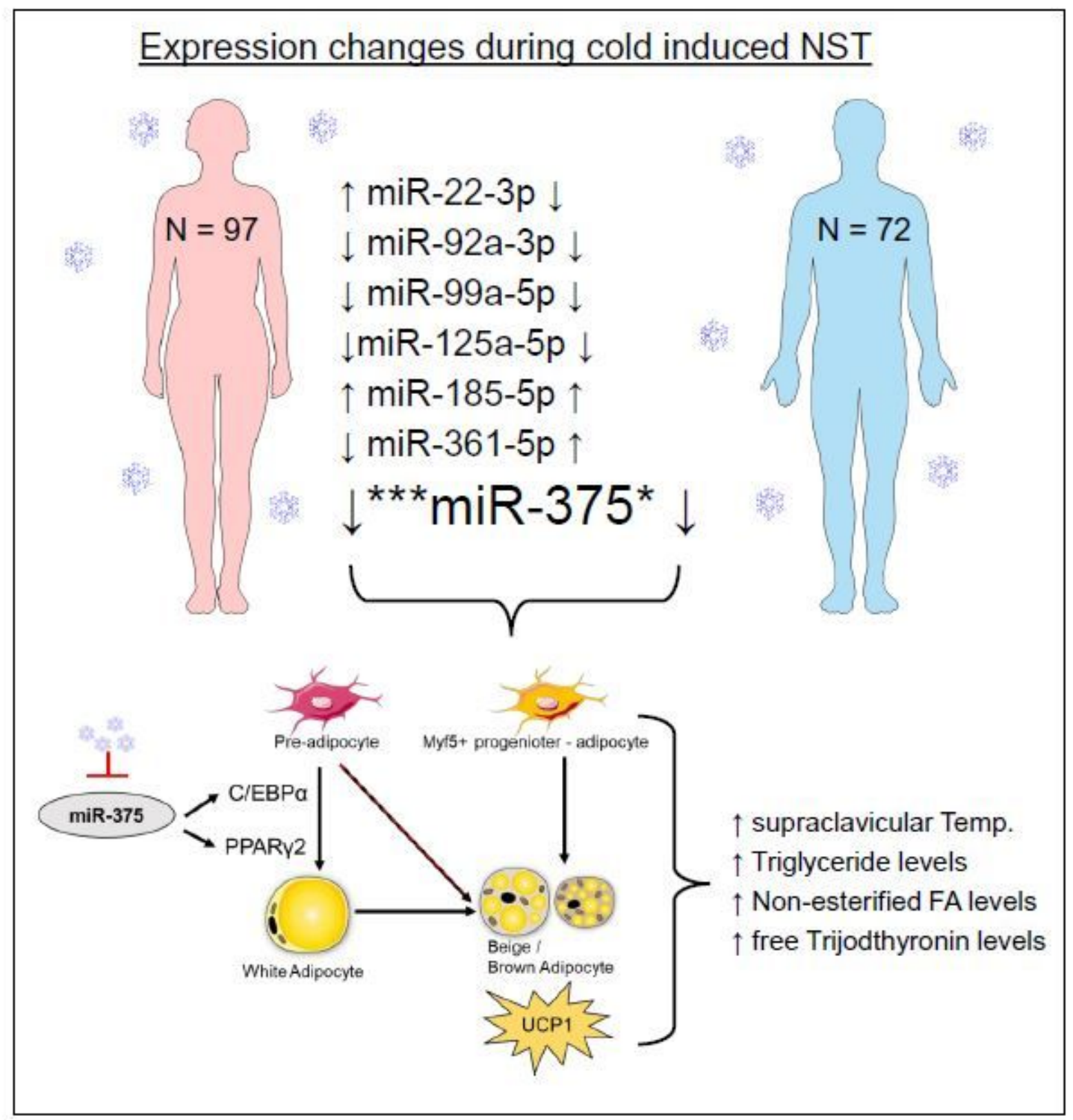

\section{Figure 4}

Summarized findings miR-375 changes in the serum of 97 female and 72 male healthy individuals before and after cold exposure (CE). miR-375 was associated with the response or non-response of CIT in a sexdependent manner.

\section{Supplementary Files}

This is a list of supplementary files associated with this preprint. Click to download.

- SupplementalmaterialmiRNA375CEfinal.pdf 